\title{
Three-dimensional extended Lifshitz, Schrödinger and Newton-Hooke supergravity
}

\author{
Nese Ozdemir, Mehmet Ozkan and Utku Zorba \\ Department of Physics, Istanbul Technical University, \\ Maslak 34469 Istanbul, Turkey \\ E-mail: nozdemir@itu.edu.tr, ozkanmehm@itu.edu.tr, zorba@itu.edu.tr
}

ABSTRACT: We provide a systematic analysis of three-dimensional $\mathcal{N}=2$ extended Bargmann superalgebra and its Newton-Hooke, Lifshitz and Schrödinger extensions. These algebras admit invariant non-degenerate bi-linear forms which we utilized to construct corresponding Chern-Simons supergravity actions.

KEYwords: Classical Theories of Gravity, Extended Supersymmetry, Supergravity Models

ArXiv EPrint: 1909.10745 


\section{Contents}

1 Introduction 1

2 Extended Newton-Hooke algebra and Chern-Simons actions 3

2.1 Extended Newton-Hooke gravity 5

2.2 Extended exotic Bargmann gravity 5

$\begin{array}{llll}3 & \text { Extended Newton-Hooke and exotic Bargmann supergravity } & 7\end{array}$

4 Extended Lifshitz and Schrödinger supergravity 10

$\begin{array}{ll}4.1 \text { Extended Lifshitz supergravity } & 10\end{array}$

$\begin{array}{ll}4.2 \text { Extended Schrödinger supergravity } & 12\end{array}$

5 Discussion $\quad 16$

$\begin{array}{ll}\text { A Comparison between different notations } & 17\end{array}$

B Extended $\mathcal{N}=(2,0)$ Newton-Hooke superalgebra from Lie algebra ex$\begin{array}{ll}\text { pansion } & 17\end{array}$

\section{Introduction}

Newton-Cartan gravity is the geometric reformulation of the Newtonian gravity that is described by a degenerate spatial metric $h^{\mu \nu}$, a temporal vielbein $\tau_{\mu}$ and a $\mathrm{U}(1)$ connection $m_{\mu} \cdot{ }^{1}$ This geometric setup of Newtonian gravity is realized based on two principles: (i) The geodesic equation is equivalent to the classical equation of motion of a massive particle, and (ii) The only non-vanishing component of the Riemann tensor gives rise to the Poisson equation of Newtonian gravity [1, 2]. If the Riemann tensor satisfies the Trautman [3] and the Ehlers [4] conditions, then we are led to the so-called Newtonian connection that is the only non-vanishing component of the connection is given by $\Gamma_{00}^{a}=\delta^{a b} \partial_{b} \phi$, satisfying (i). Consequently, the only non-vanishing component of the Riemann tensor becomes the desired Poisson's equation for Newtonian gravity

$$
R_{0 a 0}^{a}(\Gamma)=\nabla^{2} \phi=4 \pi G \rho,
$$

where $G$ is Newton's constant and $\rho$ is the mass density. An alternative derivation of this geometric formulation can be achieved by gauging the underlying symmetry algebra that is the Bargmann algebra [5]. The Bargmann algebra consists of the generators of spatial

\footnotetext{
${ }^{1}$ In this paper, the Greek indices $(\mu, \nu, \ldots)$ refer to the coordinate frame and labels all spacetime coordinates with $x=(t, \vec{x})$ while the Latin alphabet letters $(a, b, \ldots)$ refer to the spatial local Galilean frame.
} 
rotations $J_{a b}$, spatial translations $P_{a}$, Galilean boosts $G_{a}$, time translations $H$ and a central charge $M$, corresponding to particle mass. In the gauging procedure, one first associates each generator with a gauge field

$$
J_{a b} \rightarrow \omega_{\mu}^{a b}, \quad P_{a} \rightarrow e_{\mu}^{a}, \quad G_{a} \rightarrow \omega_{\mu}^{a}, \quad H \rightarrow \tau_{\mu}, \quad M \rightarrow m_{\mu},
$$

and imposes constraints on the curvatures of $\tau_{\mu}, e_{\mu}{ }^{a}$ and $m_{\mu}$ which connect the gauge transformations to general coordinate transformations and solve $\omega_{\mu}^{a b}$ and $\omega_{\mu}^{a}$ in terms of $e_{\mu}{ }^{a}, \tau_{\mu}$ and $m_{\mu}$, leaving the triplet $\left(e_{\mu}{ }^{a}, \tau_{\mu}, m_{\mu}\right)$ as the fundamental fields of the gauge theory formulation of Newton-Cartan gravity. Finally, one imposes two further constraints on the curvatures of $\omega_{\mu}^{a b}$ and $\omega_{\mu}{ }^{a}$ and recover the Poisson equation of Newtonian gravity and the geodesic equation for a massive particle from a gauge theory viewpoint.

In three space-time dimensions, the Einstein gravity can be established as a ChernSimons gauge theory in which case the necessary curvature constraints arise as an equation of motion [6]. This line of thinking encourages one to build up a similar three-dimensional framework for the Newton-Cartan gravity. Indeed in [7] this problem was addressed and it was shown that the three-dimensional Bargmann algebra does not admit a non-degenerate invariant bi-linear form to construct a Chern-Simons theory of gravity but an extension of the Bargmann algebra with a second central charge $S$ is necessary. The resulting algebra is called the extended Bargmann algebra and the corresponding Chern-Simons model is called the extended Bargmann gravity (EBG) [7]. The EBG is in many ways different than Newton-Cartan gravity. First of all, while the Newton-Cartan gravity dictates that only the purely time-like component of the Ricci tensor is non-zero (1.1), EBG allows all components of the Ricci tensor to be non-zero in the presence of matter coupling [8]. Second, the extended Bargmann algebra further allows a non-relativistic conformal extension and a corresponding action principle, called the extended Schrödinger gravity [9]. This extension is noteworthy as it corresponds to a conformal non-projectable Horava-Lifshitz gravity [9] through a map between the Newton-Cartan geometry and Horava-Lifshitz gravity [10]. Finally, the extended Bargmann algebra admits a supersymmetric extension and a corresponding on-shell Chern-Simons supergravity action [8]. The existence of a supersymmetric action is particularly important as it signals the possibility of an off-shell action for the extended Bargmann supergravity which can serve as a starting point to construct non-relativistic field theories on curved backgrounds by means of localization [11, 12].

The on-shell $\mathcal{N}=2$ extended Bargmann supergravity has the following field content [8]

$$
\left\{e_{\mu}{ }^{a}, \tau_{\mu}, m_{\mu}, \psi_{\mu}^{+}, \psi_{\mu}^{-}, \rho_{\mu}\right\}
$$

where the fermionic fields $\psi_{\mu}^{ \pm}$and $\rho_{\mu}$ are Majorana fermions that are associated with the fermionic generators $Q^{ \pm}$and $R$, respectively. Here, we used the $\mathcal{N}=2$ terminology as the on-shell EBG can be derived by a Lie algebra expansion of the three-dimensional $\mathcal{N}=2$ Poincaré supergravity [13]. The construction of an off-shell extended Bargmann supergravity requires the addition of auxiliary fields and the application of localization techniques requires the knowledge of off-shell matter multiplet actions of extended Bargmann superalgebra. In the relativistic context, this is most straightforwardly achieved by applying 
superconformal tensor calculus [14-17], where one first constructs superconformal models then gauge fix the redundant conformal symmetries to obtain a super-Poincaré invariant theory. In the case of non-relativistic gravity, a methodology for a conformal tensor calculus was established for the bosonic and supersymmetric conformal extension of the Galilei algebra, known as the Schrödinger algebra, in [18] and [19], respectively.

In this paper, our purpose is to take a pioneering step towards an extended Schrödinger tensor calculus by establishing the Schrödinger extension of the extended Bargmann algebra and the corresponding extended Schrödinger supergravity. Due to the map between the Newton-Cartan geometry and Horava-Lifshitz gravity, this result also corresponds to a superconformal non-projectable Horava-Lifshitz gravity. To achieve the extended Schrödinger superalgebra, we first remind the reader in section 2 about the fundamentals of the extended Bargmann algebra and its cosmological extension, known as the extended Newton-Hooke algebra. In section 3, we construct the cosmological extension of the extended Bargmann superalgebra. The cosmological extension of the algebra allows us to construct the extended Newton-Hooke and the extended exotic $\mathcal{N}=(2,0)$ Bargmann supergravity. Here, $\mathcal{N}=(2,0)$ terminology refers to the fact that the non-relativistic algebra can be understood as the Lie algebra expansion of the three-dimensional $\mathcal{N}=(2,0)$ AdS algebra. In section 4 we start adding dilatation and non-relativistic special conformal generators to the extended Bargmann algebra. In doing so, we first add the dilatations $D$ and a central charge $Y$. Perhaps unexpectedly, we found that the superalgebra can be closed by adding two $R$-symmetry generators and three fermionic generators $Q^{ \pm}$and $R$. The presence of the central charge $Y$ fixes the so-called dynamical critical exponent $z$ to $z=2$ and we establish $z=2$ extended Lifshitz supergravity. In the final step, we add the non-relativistic special conformal generator $K$ and a second central charge $Z$. The supersymmetric extension of the extended Schrödinger algebra requires two additional fermionic generators, which we call $F^{ \pm}$. With the addition of the new fermionic generators, we close the superalgebra and construct the Chern-Simons action for the extended Schrödinger gravity.

\section{Extended Newton-Hooke algebra and Chern-Simons actions}

In this first section, we briefly review the extended Newton-Hooke gravity and extended exotic Bargmann gravity as well as the underlying symmetry algebra that is the extended Newton-Hooke algebra. The Bargmann algebra consists of the generators of spatial rotations $J$, spatial translations $P_{a}$, Galilean boosts $G_{a}$, time translations $H$ and a central charge $M$. The extended Bargmann algebra extends the Bargmann algebra with a second central charge $S$ [7-9]. The non-vanishing commutation relations for the extended Bargmann algebra are given by

$$
\begin{aligned}
{\left[H, G_{a}\right] } & =-\epsilon_{a b} P^{b}, & {\left[J, P_{a}\right] } & =-\epsilon_{a b} P^{b}, \\
{\left[G_{a}, P_{b}\right] } & =\epsilon_{a b} M, & {\left[G_{a}, G_{b}\right] } & =\epsilon_{a b} S,
\end{aligned}
$$

Here, we use the conventions of [8]. For reader's convenience, we present a map between different conventions in appendix A. The cosmological extension of the extended Bargmann 
algebra requires two extra commutators

$$
\left[H, P_{a}\right]=-\frac{1}{\ell^{2}} \epsilon_{a b} G^{b}, \quad\left[P_{a}, P_{a}\right]=\frac{1}{\ell^{2}} \epsilon_{a b} S .
$$

Note that we set $\Lambda=\frac{1}{\ell^{2}}$ for future purposes, however the opposite sign is equally well to construct the cosmological bosonic models. The construction of actions for this symmetry algebra is based on the Chern-Simons action

$$
S=\frac{k}{4 \pi} \int \mathrm{S} \operatorname{Tr}\left(\mathrm{A} \wedge \mathrm{dA}+\frac{2}{3} \mathrm{~A} \wedge \mathrm{A} \wedge \mathrm{A}\right)
$$

where $k$ is the Chern-Simons coupling constant, the gauge field A represents a Lie-algebravalued one form and "STr" represents the supertrace using the non-degenerate invariant bilinear form on the Lie algebra, which turns into the standard trace for the purely bosonic models. For the extended Bargmann algebra, the gauge field $A_{\mu}$ is given by

$$
A_{\mu}=\tau_{\mu} H+e_{\mu}{ }^{a} P_{a}+\omega J+\omega_{\mu}{ }^{a} G_{a}+m_{\mu} M+s_{\mu} S .
$$

The transformation rules for these gauge fields can be found by the standard rule

$$
\delta A_{\mu}^{A}=\partial_{\mu} \epsilon^{A}+f_{B C}^{A} \epsilon^{C} A_{\mu}^{B},
$$

where $\epsilon^{A}$ is the relevant gauge parameter and $f_{B C}{ }^{A}$ are the structure constants. The covariant curvatures are given by

$$
\begin{aligned}
R_{\mu \nu}(H) & =2 \partial_{[\mu} \tau_{\nu]}, \\
R_{\mu \nu}{ }^{a}(P) & =2 \partial_{[\mu} e_{\nu]}^{a}+2 \epsilon^{a b} \omega_{[\mu} e_{\nu] b}-2 \epsilon^{a b} \omega_{[\mu b} \tau_{\nu]}, \\
R_{\mu \nu}(J) & =2 \partial_{[\mu} \omega_{\nu]}, \\
R_{\mu \nu}^{a}(G) & =2 \partial_{[\mu} \omega_{\nu]}^{a}+2 \epsilon^{a b} \omega_{[\mu} \omega_{\nu] b}+\frac{2}{\ell^{2}} \epsilon^{a b} \tau_{[\mu} e_{\nu] b}, \\
R_{\mu \nu}(M) & =2 \partial_{[\mu} m_{\nu]}+2 \epsilon_{a b} \omega_{[\mu}^{a} e_{\nu]}^{b} \\
R_{\mu \nu}(S) & =2 \partial_{[\mu} s_{\nu]}+\epsilon_{a b} \omega_{[\mu}^{a} \omega_{\nu]}^{b}+\frac{1}{\ell^{2}} \epsilon_{a b} e_{[\mu}^{a} e_{\nu]}^{b} .
\end{aligned}
$$

Note that for $\ell \rightarrow \infty$, these curvatures become the curvatures of the extended Bargmann algebra.

As mentioned, the construction of a Chern-Simons action requires non-degenerate invariant bilinear forms which can simply be found by constructing a Casimir operator $C$. In the case of extended Newton-Hooke algebra the following bilinears are of interest

- $\left(P_{a}, G_{b}\right)=\delta_{a b}, \quad(H, S)=-1, \quad(J, M)=-1$

- $\left(P_{a}, P_{b}\right)=\frac{1}{\ell} \delta_{a b}, \quad\left(G_{a}, G_{b}\right)=\ell \delta_{a b}, \quad(H, M)=-\frac{1}{\ell}, \quad(J, S)=-\ell$,

In the first case, the cosmological constant does not appear in the bilinears hence it can also be used to construct the extended Bargmann gravity without a cosmological constant. On the other hand, the second set only exists in the presence of a cosmological constant. A more detailed discussion on the invariant bilinear forms, including the degenerate ones in context of extended Bargmann algebra, can be found in [9]. 


\subsection{Extended Newton-Hooke gravity}

For the construction of the extended Newton-Hooke gravity we utilize the first set of invariant bilinear forms

$$
\left(P_{a}, G_{b}\right)=\delta_{a b}, \quad(H, S)=-1, \quad(J, M)=-1,
$$

and the structure constants of the extended Newton-Hooke algebra (2.1) and (2.2). In this case, the Chern-Simons action (2.3) read

$$
S=\frac{k}{4 \pi} \int d^{3} x \epsilon^{\mu \nu \rho}\left(e_{\mu}{ }^{a} R_{\nu \rho a}(G)-\tau_{\mu} R_{\nu \rho}(S)-m_{\mu} R_{\nu \rho}(J)+\frac{2}{\ell^{2}} \epsilon_{a b} \tau_{\mu} e_{\nu}{ }^{a} e_{\rho}^{b}\right),
$$

where the curvatures are as defined in (2.6). For $\ell \rightarrow \infty$, this action coincides with the extended Bargmann gravity of [7-9]. Interestingly, the action for the extended NewtonHooke gravity can also be obtained by a contraction procedure, mimicking the InonuWigner contraction $[8] .^{2}$ In order to do so, one needs to consider the cosmological EinsteinHilbert action plus a Chern-Simons action for two abelian gauge fields $Z_{1 \mu}$ and $Z_{2 \mu}$ with an off-diagonal coupling [8]

$$
S=\frac{k \omega}{4 \pi} \int d^{3} x \epsilon^{\mu \nu \rho}\left(\eta_{A B} E_{\mu}{ }^{A} R_{\nu \rho}{ }^{B}(\Omega)+\frac{1}{3 L^{2}} \epsilon_{A B C} E_{\mu}{ }^{A} E_{\nu}{ }^{B} E_{\rho}{ }^{C}+2 Z_{1 \mu} \partial_{\nu} Z_{2 \rho}\right),
$$

where

$$
R_{\mu \nu}^{A}(\Omega)=2 \partial_{[\mu} \Omega_{\nu]}^{A}+\epsilon^{A B C} \Omega_{\mu B} \Omega_{\nu C} .
$$

In terms of the non-relativistic fields and parameters, the relativistic fields and parameters are $[8]$

$$
\begin{array}{llrl}
E_{\mu}{ }^{a} & =e_{\mu}{ }^{a}, & E_{\mu}{ }^{0}=\omega \tau_{\mu}+\frac{1}{2 \omega} m_{\mu}, & \Omega_{\mu}{ }^{0}=\omega_{\mu}+\frac{1}{2 \omega^{2}} s_{\mu}, \quad L=\omega \ell, \\
\Omega_{\mu}{ }^{a}=\frac{1}{\omega} \omega_{\mu}{ }^{a}, & Z_{1 \mu}=\omega \tau_{\mu}-\frac{1}{2 \omega} m_{\mu}, & Z_{2 \mu}=\omega_{\mu}-\frac{1}{2 \omega^{2}} s_{\mu} .
\end{array}
$$

The off-diagonal coupling of the vector fields are essential for removing the $\omega^{2}$ divergencies. In the $\omega \rightarrow \infty$ limit, the action (2.9) is precisely recover the extended Newton-Hooke gravity action (2.8).

\subsection{Extended exotic Bargmann gravity}

In three dimensions there is an alternative action that gives rise to the Einstein's equations in the presence of a cosmological constant, known as the exotic Einstein-Hilbert action $[6,22]$

$$
S=\frac{k L \omega}{2 \pi} \int d^{3} x \epsilon^{\mu \nu \rho}\left(\Omega_{\mu}{ }^{A} \partial_{\nu} \Omega_{\rho A}+\frac{1}{3} \epsilon_{A B C} \Omega_{\mu}{ }^{A} \Omega_{\nu}{ }^{B} \Omega_{\rho}{ }^{C}+\frac{1}{2 L^{2}} E_{\mu}{ }^{A} R_{\nu \rho A}(P)\right) .
$$

\footnotetext{
${ }^{2}$ Extended Bargmann algebra can also be obtained by the Inönü-Wigner contraction of the Poincaré $\otimes$ $\mathrm{U}(1)^{2}$ algebra, see [20, 21].
} 
This indicates that there might be an extended "exotic" Bargmann gravity following the contraction procedure introduced in (2.11). As the exotic theory has diagonal coupling amongst the gauge fields of the Poincaré algebra, the vector fields that are neccessary to cancel out the $\omega^{2}$ divergences should also appear with a diagonal coupling. Thus, we consider the exotic Einstein-Hilbert action plus a diagonal Chern-Simon action for two abelian gauge fields

$$
\begin{aligned}
S=\frac{k L \omega}{2 \pi} \int d^{3} x \epsilon^{\mu \nu \rho}\left(\Omega_{\mu}{ }^{A} \partial_{\nu} \Omega_{\rho A}+\frac{1}{3} \epsilon_{A B C} \Omega_{\mu}{ }^{A} \Omega_{\nu}{ }^{B} \Omega_{\rho}{ }^{C}\right. \\
\\
\left.+\frac{1}{2 L^{2}} E_{\mu}{ }^{A} R_{\nu \rho A}(P)+\frac{1}{L^{2}} Z_{1 \mu} \partial_{\nu} Z_{1 \rho}+Z_{2 \mu} \partial_{\nu} Z_{2 \rho}\right),
\end{aligned}
$$

where

$$
R_{\mu \nu}^{A}(P)=2 \partial_{[\mu} E_{\nu]}^{A}+2 \epsilon^{A B C} \Omega_{[\mu B} E_{\nu] C} .
$$

Using the expressions in (2.11) in the action (2.13), we find that the extended exotic Bargmann gravity is given by

$$
S=\frac{k \ell}{4 \pi} \int d^{3} x \epsilon^{\mu \nu \rho}\left(\omega_{\mu}{ }^{a} R_{\nu \rho a}(G)-2 s_{\mu} R_{\nu \rho}(J)+\frac{1}{\ell^{2}}\left(e_{\mu}{ }^{a} R_{\nu \rho a}(P)-2 m_{\mu} R_{\nu \rho}(H)\right)\right) .
$$

At this stage, it is worthwhile to discuss the "exotic" terminology in the context of relativistic and non-relativistic gravity. In the relativistic context, the fundamental fields $E^{a}$ and $\Omega^{a b}$ are parity even, thus the dual spin connection, $\Omega^{a}$ is parity odd. As a result the Einstein-Hilbert action (2.9) is parity even while the exotic Einstein-Hilbert action (2.12) is parity odd. Here, the exotic terminology emphasizes the fact that although the exotic Einstein-Hilbert action is of odd-parity, it give rise to parity even equations of motion. In the non-relativistic context, we have the following parity assignment for the gauge fields

$$
\text { Even: }\left\{\tau_{\mu}, \quad e_{\mu}^{a}, m_{\mu}\right\}, \quad \text { Odd: }\left\{\omega_{\mu}, \omega_{\mu}^{a}, s_{\mu}\right\} \text {. }
$$

In this case, the extended Newton-Hooke gravity is parity even while the extended exotic Bargmann gravity is parity odd. As both of these theories have parity even equations of motion, we use the "exotic" terminology for the action (2.15). It is also possible to obtain the extended exotic Bargmann gravity by using the second set of bilinear forms [9]

$$
\left(P_{a}, P_{b}\right)=\frac{1}{\ell^{2}} \delta_{a b}, \quad\left(G_{a}, G_{b}\right)=\delta_{a b}, \quad(H, M)=-\frac{1}{\ell^{2}}, \quad(J, S)=-1,
$$

and tracing the Chern-Simons action. The resulting action precisely coincide with the extended exotic Bargmann gravity (2.15).

Although the field equations for the extended Newton-Hooke gravity (2.8) and the extended exotic Bargmann gravity (3.8) are the same, these two models differ dramatically when we consider the matter couplings. In particular, let us first take a closer look at the $s_{\mu}, \omega_{\mu}{ }^{a}$ and $\omega_{\mu}$ equations for the extended Newton-Hooke gravity

$$
R_{\mu \nu}(H)=0, \quad R_{\mu \nu}^{a}(P)=0, \quad R_{\mu \nu}(M)=0 .
$$


The first equation implies that this model is defined on non-relativistic space-times with torsionless Newton-Cartan geometry while the last two equations give rise to composite expressions for $\omega_{\mu}$ and $\omega_{\mu}{ }^{a}$. The torsionless condition is imposed employing $s_{\mu}$ equation, which suggests that one needs to find a matter content that transforms non-trivially under $S$-transformations to enable torsional non-relativistic background geometries. On the other hand, the same torsion-free constraint is imposed by the $m_{\mu}$ equation in the case of extended exotic Bargmann gravity. Accordingly one needs to find a matter content that transforms non-trivially under $M$-transformations to include torsion. As opposed to the extended Bargmann gravity, this can be simply achieved, for instance, by coupling a complex scalar field to extended exotic Bargmann gravity. Consequently, extended exotic Bargmann gravity provides a more useful setup to study three-dimensional torsional non-relativistic geometries.

\section{Extended Newton-Hooke and exotic Bargmann supergravity}

The $\mathcal{N}=(2,0)$ supersymmetric extension of the extended Newton-Hooke algebra, $(2.1)$ and (2.2), requires three supercharges $Q_{\alpha}^{ \pm}$and $R_{\alpha}(\alpha=1,2)$ that are all Majorana spinors. It furthermore requires two extra bosonic generators $U_{1}$ and $U_{2}$. Both $U_{1}$ and $U_{2}$ act nontrivially on the spinors $Q^{ \pm}$and $R$ in the presence of the cosmological constant and they become central when cosmological constant vanishes. The $\mathcal{N}=(2,0)$ extended NewtonHooke superalgebra has the following non-vanising $[B, B]$ and $[B, F]$ commutators

$$
\begin{aligned}
& {\left[H, G_{a}\right]=-\epsilon_{a b} P^{b},} \\
& {\left[J, P_{a}\right]=-\epsilon_{a b} P^{b},} \\
& {\left[G_{a}, P_{b}\right]=\epsilon_{a b} M,} \\
& {\left[G_{a}, G_{b}\right]=\epsilon_{a b} S,} \\
& {\left[H, P_{a}\right]=-\frac{1}{\ell^{2}} \epsilon_{a b} G^{b},} \\
& {\left[J, Q^{ \pm}\right]=-\frac{1}{2} \gamma_{0} Q^{ \pm},} \\
& {\left[S, Q^{+}\right]=-\frac{1}{2} \gamma_{0} R,} \\
& {\left[G_{a}, Q^{+}\right]=-\frac{1}{2} \gamma_{a} Q^{-},} \\
& {\left[P_{a}, Q^{+}\right]=-\frac{1}{2 \ell} \gamma_{a} Q^{-},} \\
& {\left[P_{a}, Q^{-}\right]=-\frac{1}{2 \ell} \gamma_{a} R,} \\
& {[H, R]=-\frac{1}{2 \ell} \gamma_{0} R,} \\
& {\left[U_{1}, R\right]=-\frac{1}{\ell} \gamma_{0} R,} \\
& {\left[M, Q^{+}\right]=-\frac{1}{2 \ell} \gamma_{0} R,} \\
& {\left[U_{2}, Q^{+}\right]=-\frac{1}{\ell} \gamma_{0} R \text {. }} \\
& {\left[J, G_{a}\right]=-\epsilon_{a b} G^{b},} \\
& {\left[P_{a}, P_{b}\right]=\frac{1}{\ell^{2}} \epsilon_{a b} S,} \\
& {[J, R]=-\frac{1}{2} \gamma_{0} R,} \\
& {\left[G_{a}, Q^{-}\right]=-\frac{1}{2} \gamma_{a} R,} \\
& {\left[H, Q^{ \pm}\right]=-\frac{1}{2 \ell} \gamma_{0} Q^{ \pm},} \\
& {\left[U_{1}, Q^{ \pm}\right]=\mp \frac{1}{\ell} \gamma_{0} Q^{ \pm},}
\end{aligned}
$$

while the non-vanishing $\{F, F\}$ anti-commutators are given by

$$
\begin{aligned}
& \left\{Q_{\alpha}^{+}, Q_{\beta}^{+}\right\}=\left(\gamma_{0} C^{-1}\right)_{\alpha \beta} H+\frac{1}{\ell}\left(\gamma_{0} C^{-1}\right)_{\alpha \beta} J-\left(\gamma_{0} C^{-1}\right)_{\alpha \beta} U_{1}, \\
& \left\{Q_{\alpha}^{+}, Q_{\beta}^{-}\right\}=-\left(\gamma_{a} C^{-1}\right)_{\alpha \beta} P^{a}-\frac{1}{\ell}\left(\gamma_{a} C^{-1}\right)_{\alpha \beta} G^{a}, \\
& \left\{Q_{\alpha}^{+}, R_{\beta}\right\}=\left(\gamma_{0} C^{-1}\right)_{\alpha \beta} M+\frac{1}{\ell}\left(\gamma_{0} C^{-1}\right)_{\alpha \beta} S-\left(\gamma_{0} C^{-1}\right)_{\alpha \beta} U_{2}, \\
& \left\{Q_{\alpha}^{-}, Q_{\beta}^{-}\right\}=\left(\gamma_{0} C^{-1}\right)_{\alpha \beta} M+\frac{1}{\ell}\left(\gamma_{0} C^{-1}\right)_{\alpha \beta} S+\left(\gamma_{0} C^{-1}\right)_{\alpha \beta} U_{2} .
\end{aligned}
$$


The appearence of the extra bosonic generators $U_{1,2}$ can be understood as the Lie algebra expansion of the $R$-symmetry generator of the $\mathcal{N}=(2,0)$ AdS superalgebra, which we defer the details to the appendix B. Here, our splitting of the indices of gamma matrices into its temporal and the spatial components as well as our treatment to gamma-matrices in general follow from [23]. As in the bosonic case, the extended Newton-Hooke algebra admit two distinct non-degenerate bilinear forms. In the first case, it is given by

$$
\begin{aligned}
& \left(P_{a}, G_{b}\right)=\delta_{a b} \\
& (H, S)=-1, \\
& \left(Q_{\alpha}^{+}, R_{\beta}\right)=2\left(C^{-1}\right)_{\alpha \beta}, \quad\left(Q_{\alpha}^{-}, Q_{\beta}^{-}\right)=2\left(C^{-1}\right)_{\alpha \beta}, \\
& (J, M)=-1, \quad\left(U_{1}, U_{2}\right)=\frac{2}{\ell},
\end{aligned}
$$

Taking the gauge field to be

$$
\begin{aligned}
A_{\mu}= & \tau_{\mu} H+e_{\mu}{ }^{a} P_{a}+\omega_{\mu} J+\omega_{\mu}{ }^{a} G_{a}+m_{\mu} M+s_{\mu} S+r_{1 \mu} U_{1}+r_{2 \mu} U_{2} \\
& +\bar{\psi}_{\mu}^{+} Q^{+}+\bar{\psi}_{\mu}^{-} Q^{-}+\bar{\rho}_{\mu} R,
\end{aligned}
$$

we can construct a Chern-Simons action by using the invariant bilinear form (3.3). The resulting theory is the extended Newton-Hooke supergravity. This action is given by

$$
\begin{aligned}
S=\frac{k}{4 \pi} \int d^{3} x \varepsilon^{\mu \nu \rho}\left(e_{\mu}{ }^{a} R_{\nu \rho a}(G)-\tau_{\mu} R_{\nu \rho}(S)-m_{\mu} R_{\nu \rho}(J)+\frac{2}{\ell^{2}} \epsilon_{a b} \tau_{\mu} e_{\nu}{ }^{a} e_{\rho}{ }^{b}\right. \\
\left.+\frac{4}{\ell} r_{\mu \mu} \partial_{\nu} r_{2 \rho}+\bar{\psi}_{\mu}^{+} R_{\nu \rho}(R)+\bar{\rho}_{\mu} R_{\nu \rho}\left(Q^{+}\right)+\bar{\psi}_{\mu}^{-} R_{\nu \rho}\left(Q^{-}\right)\right) .
\end{aligned}
$$

The bosonic part of this action, excluding the $R$-symmetry part, matches with the extended Newton-Hooke action given in [9]. Here, the bosonic curvatures are as given in (2.6) while the fermionic curvatures are given as

$$
\begin{aligned}
R_{\mu \nu}\left(Q^{+}\right)= & 2 \partial_{[\mu} \psi_{\nu]}^{+}+\omega_{[\mu} \gamma_{0} \psi_{\nu]}^{+}+\frac{1}{\ell} \tau_{[\mu} \gamma_{0} \psi_{\nu]}^{+}+\frac{2}{\ell} r_{1[\mu} \gamma_{0} \psi_{\nu]}^{+}, \\
R_{\mu \nu}\left(Q^{-}\right)= & 2 \partial_{[\mu} \psi_{\nu]}^{-}+\omega_{[\mu} \gamma_{0} \psi_{\nu]}^{-}+\omega_{[\mu}^{a} \gamma_{a} \psi_{\nu]}^{+}+\frac{1}{\ell} \tau_{[\mu} \gamma_{0} \psi_{\nu]}^{-}+\frac{1}{\ell} e_{[\mu}^{a} \gamma_{a} \psi_{\nu]}^{+}-\frac{2}{\ell} r_{1[\mu} \gamma_{0} \psi_{\nu]}^{-}, \\
R_{\mu \nu}(R)= & 2 \partial_{[\mu} \rho_{\nu]}+\omega_{[\mu} \gamma_{0} \rho_{\nu]}+\omega_{[\mu}^{a} \gamma_{a} \psi_{\nu]}^{-}+s_{[\mu} \gamma_{0} \psi_{\nu]}^{+}+\frac{1}{\ell} \tau_{[\mu} \gamma_{0} \rho_{\nu]}+\frac{1}{\ell} m_{[\mu} \gamma_{0} \psi_{\nu]}^{+} \\
& +\frac{2}{\ell} r_{1[\mu} \gamma_{0} \rho_{\nu]}+\frac{1}{\ell} e_{[\mu}^{a} \gamma_{a} \psi_{\nu]}^{-}+\frac{2}{\ell} r_{2[\mu} \gamma_{0} \psi_{\nu]}^{+} .
\end{aligned}
$$

In the presence of a cosmological constant, there is a second non-degerate invariant bilinear form that is given by

$$
\begin{aligned}
& \left(P_{a}, P_{b}\right)=\frac{1}{\ell} \delta_{a b}, \quad\left(G_{a}, G_{b}\right)=\ell \delta_{a b}, \quad(H, M)=-\frac{1}{\ell}, \quad(J, S)=-\ell, \\
& \left(U_{1}, U_{2}\right)=\frac{2}{\ell}, \quad\left(Q_{\alpha}^{+}, R_{\beta}\right)=2\left(C^{-1}\right)_{\alpha \beta}, \quad\left(Q_{\alpha}^{-}, Q_{\beta}^{-}\right)=2\left(C^{-1}\right)_{\alpha \beta},
\end{aligned}
$$

In this case, we obtain the "exotic" extended Bargmann supergravity

$$
\begin{aligned}
S=\frac{k \ell}{4 \pi} \int d^{3} x \epsilon^{\mu \nu \rho}( & \omega_{\mu}{ }^{a} R_{\nu \rho a}(G)-2 s_{\mu} R_{\nu \rho}(J)+\frac{1}{\ell^{2}} e_{\mu}{ }^{a} R_{\nu \rho a}(P)-\frac{2}{\ell^{2}} \tau_{\mu} R_{\nu \rho}(M) \\
+ & \left.\frac{8}{\ell^{2}} r_{2 \mu} \partial_{\nu} r_{1 \rho}+\frac{2}{\ell} \bar{\psi}_{\mu}^{+} R_{\nu \rho}(R)+\frac{2}{\ell} \bar{\rho}_{\mu} R_{\nu \rho}\left(Q^{+}\right)+\frac{2}{\ell} \bar{\psi}_{\mu}^{-} R_{\nu \rho}\left(Q^{-}\right)\right) .
\end{aligned}
$$


The bosonic part of this action, excluding the $R$-symmetry part, matches with the extended exotic Bargmann action given in [9]. The extended Newton-Hooke (3.5) and the exotic extended Bargmann gravity (3.8) are invraiant under the following supersymmetry transformation rules

$$
\begin{aligned}
\delta \tau_{\mu}= & -\bar{\epsilon}^{+} \gamma_{0} \psi_{\mu}^{+}, \\
\delta e_{\mu}{ }^{a}= & \bar{\epsilon}^{+} \gamma^{a} \psi_{\mu}^{-}+\bar{\epsilon}^{-} \gamma^{a} \psi_{\mu}^{+}, \\
\delta \omega_{\mu}{ }^{a}= & \frac{1}{\ell}\left(\bar{\epsilon}^{+} \gamma^{a} \psi_{\mu}^{-}+\bar{\epsilon}^{-} \gamma^{a} \psi_{\mu}^{+}\right) \\
\delta \omega_{\mu}= & -\frac{1}{\ell} \bar{\epsilon}^{+} \gamma_{0} \psi_{\mu}^{+}, \\
\delta m_{\mu}= & -\bar{\epsilon}^{-} \gamma_{0} \psi_{\mu}^{-}-\bar{\epsilon}^{+} \gamma_{0} \rho_{\mu}-\bar{\eta} \gamma_{0} \psi_{\mu}^{+}, \\
\delta s_{\mu}= & -\frac{1}{\ell}\left(\bar{\epsilon}^{-} \gamma_{0} \psi_{\mu}^{-}+\bar{\epsilon}^{+} \gamma_{0} \rho_{\mu}+\bar{\eta} \gamma_{0} \psi_{\mu}^{+}\right) \\
\delta r_{1 \mu}= & \bar{\epsilon}^{+} \gamma_{0} \psi_{\mu}^{+}, \\
\delta r_{2 \mu}= & -\bar{\epsilon}^{-} \gamma_{0} \psi_{\mu}^{-}+\bar{\epsilon}^{+} \gamma_{0} \rho_{\mu}+\bar{\eta} \gamma_{0} \psi_{\mu}^{+}, \\
\delta \psi_{\mu}^{+}= & \partial_{\mu} \epsilon^{+}+\frac{1}{2} \omega_{\mu} \gamma_{0} \epsilon^{+}+\frac{1}{2 \ell} \tau_{\mu} \gamma_{0} \epsilon^{+}+\frac{1}{\ell} r_{1 \mu} \gamma_{0} \epsilon^{+}, \\
\delta \psi_{\mu}^{-}= & \partial_{\mu} \epsilon^{-}+\frac{1}{2} \omega_{\mu} \gamma_{0} \epsilon^{-}+\frac{1}{2} \omega_{\mu}^{a} \gamma_{a} \epsilon^{+}+\frac{1}{2 \ell} \tau_{\mu} \gamma_{0} \epsilon^{-}+\frac{1}{2 \ell} e_{\mu}^{a} \gamma_{a} \epsilon^{+}-\frac{1}{\ell} r_{1 \mu} \gamma_{0} \epsilon^{-}, \\
\delta \rho_{\mu}= & \partial_{\mu} \eta+\frac{1}{2} \omega_{\mu} \gamma_{0} \eta+\frac{1}{2} \omega_{\mu}^{a} \gamma_{a} \epsilon^{-}+\frac{1}{2} s_{\mu} \gamma_{0} \epsilon^{+}+\frac{1}{2 \ell} \tau_{\mu} \gamma_{0} \eta+\frac{1}{2 \ell} m_{\mu} \gamma_{0} \epsilon^{+} \\
& +\frac{1}{2 \ell} e_{\mu}^{a} \gamma_{a} \epsilon^{-}+\frac{1}{\ell} r_{1 \mu} \gamma_{0} \eta+\frac{1}{\ell} r_{2 \mu} \gamma_{0} \epsilon^{+},
\end{aligned}
$$

where $\epsilon^{ \pm}$and $\eta$ are the the parameters of the local $Q^{ \pm}$and $R$ transformations, respectively. In the supersymmetric extension of the extended Newton-Hooke gravity (or extended exotic Bargmann gravity), the curvature constraints that are given by the $s_{\mu}, \omega_{\mu}{ }^{a}, \omega_{\mu}$ (or $\left.m_{\mu}, e_{\mu}{ }^{a}, \tau_{\mu}\right)$ are given by

$$
R_{\mu \nu}(H)=0, \quad R_{\mu \nu}{ }^{a}(P)=0, \quad R_{\mu \nu}(M)=0,
$$

where

$$
\begin{aligned}
& R_{\mu \nu}(H)=2 \partial_{[\mu} \tau_{\nu]}+\bar{\psi}_{\mu} \gamma_{0} \psi_{\nu}, \\
& R_{\mu \nu}{ }^{a}(P)=2 \partial_{[\mu} e_{\nu]}^{a}+2 \epsilon^{a b} \omega_{[\mu} e_{\nu] b}-2 \epsilon^{a b} \omega_{[\mu b} \tau_{\nu]}-2 \bar{\psi}_{[\mu}^{+} \gamma^{a} \psi_{\nu]}^{-}, \\
& R_{\mu \nu}(M)=2 \partial_{[\mu} m_{\nu]}+2 \epsilon_{a b} \omega_{[\mu}^{a} e_{\nu]}^{b}+2 \bar{\psi}_{[\mu}^{+} \gamma_{0} \rho_{\nu]}+\bar{\psi}_{\mu}^{-} \gamma_{0} \psi_{\nu}^{-} .
\end{aligned}
$$

In this case, first equation imply a fermionic temporal torsion while the last two equations gives rise to composite expressions for $\omega_{\mu}$ and $\omega_{\mu}{ }^{a}$ that includes a fermionic part. The back substitution of these composite expressions into the action (2.8) (or (3.8)) lead to quartic fermionic terms in the action. 


\section{Extended Lifshitz and Schrödinger supergravity}

In this section, we take our final step towards the completion of the realm of threedimensional $\mathcal{N}=2$ extended Bargmann supergravity. The missing pieces are models of supergravity with more symmetries such as dilatations and non-relativistic special conformal symmetry. In the first subsection, we show that it is possible to extend the extended Bargmann algebra with dilatations and a central charge, $Y$, giving rise to the extended Lifshitz superalgebra and consequently the extended Lifshitz supergravity. We then proceed with the full Schrödinger superalgebra and construct a Chern-Simons action as an extended Schrödinger supergravity.

\subsection{Extended Lifshitz supergravity}

The inclusion of dilatations $(D)$ in the extended Bargmann algebra (2.1) requires an additional central charge $(Y)$ in order to have a non-degenerate metric. The non-vanishing commutation relations for this algebra are given by

$$
\begin{aligned}
& {\left[H, G_{a}\right]=-\epsilon_{a b} P^{b}, \quad\left[J, P_{a}\right]=-\epsilon_{a b} P^{b}, \quad\left[J, G_{a}\right]=-\epsilon_{a b} G^{b},} \\
& {\left[P_{a}, G_{b}\right]=\epsilon_{a b} M+\delta_{a b} Y, \quad\left[G_{a}, G_{b}\right]=\epsilon_{a b} S, \quad[D, S]=2 S,} \\
& {\left[D, G_{a}\right]=G_{a}, \quad\left[D, P_{a}\right]=-P_{a}, \quad[D, H]=-2 H,} \\
& {[H, S]=-2 Y,}
\end{aligned}
$$

We refer to this algebra as the extended extended Lifshitz algebra. Note here that the Lifshitz symmetry usually allow the scaling dimension of the temporal translations $H$ to be arbitrary, which is referred to as the dynamical critical exponent $z$. However, the inclusion of the central charge $Y$ fixes the scaling dimension of $H$ to $z=2$. This algebra allows the following non-degenerate invariant bi-linear form

$$
\left(P_{a}, G_{b}\right)=\delta_{a b}, \quad(S, H)=-1, \quad(M, J)=-1, \quad(Y, D)=-1 .
$$

Taking the gauge field to be

$$
A_{\mu}=\tau_{\mu} H+e_{\mu}^{a} P_{a}+\omega_{\mu} J+\omega_{\mu}^{a} G_{a}+m_{\mu} M+s_{\mu} S+b_{\mu} D+y_{\mu} Y,
$$

we can use to construct a Chern-Simons action for the extended Lifshitz algebra

$$
\begin{aligned}
S=\frac{k}{4 \pi} \int d^{3} x \varepsilon^{\mu \nu \rho}\left(\omega_{\mu}{ }^{a} R_{\nu \rho a}(P)-2 s_{\mu} R_{\nu \rho}(\right. & H)-2 m_{\mu} R_{\nu \rho}(J) \\
& \left.+e_{\mu}{ }^{a} R_{\nu \rho a}(G)-2 y_{\mu} R_{\nu \rho}(D)\right) .
\end{aligned}
$$

Here, the curvatures of the gauge fields are given by

$$
\begin{aligned}
R_{\nu \rho}^{a}(P) & =2 \partial_{[\mu} e_{\nu]}^{a}+2 \epsilon^{a b} \omega_{[\mu} e_{\nu] b}-2 \epsilon^{a b} \omega_{[\mu b} \tau_{\nu]}-2 b_{[\mu} e_{\nu]}^{a}, \\
R_{\nu \rho}^{a}(G) & =2 \partial_{[\mu} \omega_{\nu]}^{a}+2 \epsilon^{a b} \omega_{[\mu} \omega_{\nu] b}+2 b_{[\mu} \omega_{\nu]}^{a} \\
R_{\nu \rho}(H) & =2 \partial_{[\mu} \tau_{\nu]}-4 b_{[\mu} \tau_{\nu]} \\
R_{\nu \rho}(J) & =2 \partial_{[\mu} \omega_{\nu]} \\
R_{\nu \rho}(D) & =2 \partial_{[\mu} b_{\nu]} .
\end{aligned}
$$


As the non-relativistic special conformal symmetry is absent, the supersymmetric extension of the extended Lifshitz algebra does not require a special supersymmetry generator but the three fermionic generators $Q^{ \pm}$and $R$ that we introduced in the previous sections are sufficient. The inclusion of the generators $U_{1,2}$ is still necessary, but while $U_{1}$ acts non-trivially on the fermionic generators, $U_{2}$ is a central charge in the extended Lifshitz superalgebra. With the assistance of the computer algebra program Cadabra [24, 25], we found that the non-vanishing $[B, B]$ commutators of the extended Lifshitz superalgebra are given by (4.1) while the non-vanishing $[B, F]$ commutators are

$$
\begin{aligned}
{\left[J, Q^{ \pm}\right] } & =-\frac{1}{2} \gamma_{0} Q^{ \pm}, & {[J, R] } & =-\frac{1}{2} \gamma_{0} R, & {\left[G_{a}, Q^{+}\right] } & =-\frac{1}{2} \gamma_{a} Q^{-}, \\
{\left[G_{a}, Q^{-}\right] } & =-\frac{1}{2} \gamma_{a} R, & {\left[U_{1}, Q^{ \pm}\right] } & = \pm \gamma_{0} Q^{ \pm}, & {\left[U_{1}, R\right] } & =\gamma_{0} R, \\
{\left[D, Q^{+}\right] } & =-Q^{+}, & {[D, R] } & =R, & {\left[S, Q^{+}\right] } & =-\frac{1}{2} \gamma_{0} R
\end{aligned}
$$

Finally, the non-vanishing $\{F, F\}$ anti-commutators are given by

$$
\begin{aligned}
& \left\{Q_{\alpha}^{+}, Q_{\beta}^{+}\right\}=\left(\gamma_{0} C^{-1}\right)_{\alpha \beta} H \\
& \left\{Q_{\alpha}^{+}, Q_{\beta}^{-}\right\}=-\left(\gamma_{a} C^{-1}\right)_{\alpha \beta} P^{a}, \\
& \left\{Q_{\alpha}^{-}, Q_{\beta}^{-}\right\}=\left(\gamma_{0} C^{-1}\right)_{\alpha \beta} M-\left(\gamma_{0} C^{-1}\right)_{\alpha \beta} U_{2}, \\
& \left\{Q_{\alpha}^{+}, R_{\beta}\right\}=\left(\gamma_{0} C^{-1}\right)_{\alpha \beta} M+2 C_{\alpha \beta} Y+\left(\gamma_{0} C^{-1}\right)_{\alpha \beta} U_{2} .
\end{aligned}
$$

The construction of a Chern-Simons action for the extended Lifshitz supergravity can be achieved by using the following non-degenerate invariant bi-linear form

$$
\begin{aligned}
\left(P_{a}, G_{b}\right) & =\delta_{a b}, & (S, H) & =-1, & (M, J) & =-1, \\
\left(U_{1}, U_{2}\right) & =2, & \left(Q_{\alpha}^{+}, R_{\beta}\right) & =2\left(C^{-1}\right)_{\alpha \beta}, & \left(Q_{\alpha}^{-}, Q_{\beta}^{-}\right) & \left.=2\left(C^{-1}\right)_{\alpha \beta} . D\right)=-1,
\end{aligned} \quad(Y,
$$

Taking the gauge field to be

$$
\begin{aligned}
A_{\mu}= & \tau_{\mu} H+e_{\mu}^{a} P_{a}+\omega_{\mu} J+\omega_{\mu}^{a} G_{a}+m_{\mu} M+s_{\mu} S+r_{1 \mu} U_{1} \\
& +r_{2 \mu} U_{2}+b_{\mu} D+y_{\mu} Y+\bar{\psi}_{\mu}^{+} Q^{+}+\bar{\psi}_{\mu}^{-} Q^{-}+\bar{\rho}_{\mu} R
\end{aligned}
$$

we can construct a supersymmetric action

$$
\begin{aligned}
S=\frac{k}{4 \pi} \int d^{3} x \varepsilon^{\mu \nu \rho}\left(\omega_{\mu}{ }^{a} R_{\nu \rho a}(P)+e_{\mu}{ }^{a} R_{\nu \rho a}(G)-2 s_{\mu} R_{\nu \rho}(H)-2 m_{\mu} R_{\nu \rho}(J)\right. \\
\left.-2 y_{\mu} R_{\nu \rho}(D)+4 r_{2 \mu} R_{\nu \rho}\left(U_{1}\right)+4 \bar{\rho}_{\mu} R_{\nu \rho}\left(Q^{+}\right)+2 \bar{\psi}_{\mu}^{-} R_{\nu \rho}\left(Q^{-}\right)\right) .
\end{aligned}
$$

We refer to this action as the extended Lifshitz supergravity. The supercovariant curvatures that appears in the action are given by

$$
\begin{aligned}
R_{\mu \nu}(H) & =2 \partial_{[\mu} \tau_{\nu]}-4 b_{[\mu} \tau_{\nu]}+\bar{\psi}_{\mu}^{+} \gamma_{0} \psi_{\nu}^{+} \\
R_{\mu \nu}{ }^{a}(P) & =2 \partial_{[\mu} e_{\nu]}^{a}+2 \epsilon^{a b} \omega_{[\mu} e_{\nu] b}-2 \epsilon^{a b} \omega_{[\mu b} \tau_{\nu]}-2 b_{[\mu} e_{\nu]}^{a}-2 \bar{\psi}_{[\mu}^{+} \gamma^{a} \psi_{\nu]}^{-}
\end{aligned}
$$




$$
\begin{aligned}
R_{\mu \nu}{ }^{a}(G) & =2 \partial_{[\mu} \omega_{\nu]}^{a}+2 \epsilon^{a b} \omega_{[\mu} \omega_{\nu] b}+2 b_{[\mu} \omega_{\nu]}^{a} \\
R_{\mu \nu}(J) & =2 \partial_{[\mu} \omega_{\nu]} \\
R_{\mu \nu}(D) & =2 \partial_{[\mu} b_{\nu]} \\
R_{\mu \nu}\left(U_{1}\right) & =2 \partial_{[\mu} r_{1 \nu]} \\
R_{\mu \nu}\left(Q^{+}\right) & =2 \partial_{[\mu} \psi_{\nu]}^{+}+\omega_{[\mu} \gamma_{0} \psi_{\nu]}^{+}-2 b_{[\mu} \psi_{\nu]}^{+}-2 r_{1[\mu} \gamma_{0} \psi_{\nu]}^{+}, \\
R_{\mu \nu}\left(Q^{-}\right) & =2 \partial_{[\mu} \psi_{\nu]}^{-}+\omega_{[\mu} \gamma_{0} \psi_{\nu]}^{-}+\omega_{[\mu}{ }^{a} \gamma_{a} \psi_{\nu]}^{+}+2 r_{1[\mu} \gamma_{0} \psi_{\nu]}^{-}, \\
R_{\mu \nu}(R) & =2 \partial_{[\mu} \rho_{\nu]}+\omega_{[\mu} \gamma_{0} \rho_{\nu]}+\omega_{[\mu}{ }^{a} \gamma_{a} \psi_{\nu]}^{-}+s_{[\mu} \gamma_{0} \psi_{\nu]}^{+}-2 r_{1[\mu} \gamma_{0} \rho_{\nu]}+2 b_{[\mu} \rho_{\nu]} .
\end{aligned}
$$

The extended Lifshitz supergravity is invariant under the following supersymmetry transformation rules

$$
\begin{aligned}
\delta \tau_{\mu} & =-\bar{\epsilon}^{+} \gamma_{0} \psi_{\mu}^{+}, \\
\delta e_{\mu}^{a} & =\bar{\epsilon}^{+} \gamma^{a} \psi_{\mu}^{-}+\bar{\epsilon}^{-} \gamma^{a} \psi_{\mu}^{+}, \\
\delta m_{\mu} & =-\bar{\epsilon}^{-} \gamma_{0} \psi_{\mu}^{-}-\bar{\epsilon}^{+} \gamma_{0} \rho_{\mu}-\bar{\eta} \gamma_{0} \psi_{\mu}^{+}, \\
\delta r_{2 \mu} & =\bar{\epsilon}^{-} \gamma_{0} \psi_{\mu}^{-}-\bar{\epsilon}^{+} \gamma_{0} \rho_{\mu}-\bar{\eta} \gamma_{0} \psi_{\mu}^{+}, \\
\delta y_{\mu} & =2 \bar{\eta} \psi_{\mu}^{+}-2 \bar{\epsilon}^{+} \rho_{\mu}, \\
\delta \psi_{\mu}^{+} & =\partial_{\mu} \epsilon^{+}+\frac{1}{2} \omega_{\mu} \gamma_{0} \epsilon^{+}-b_{\mu} \epsilon^{+}-r_{1 \mu} \gamma_{0} \epsilon^{+}, \\
\delta \psi_{\mu}^{-} & =\partial_{\mu} \epsilon^{-}+\frac{1}{2} \omega_{\mu} \gamma_{0} \epsilon^{-}+\frac{1}{2} \omega_{\mu}^{a} \gamma_{a} \epsilon^{+}+r_{1 \mu} \gamma_{0} \epsilon^{-}, \\
\delta \rho_{\mu} & =\partial_{\mu} \eta+\frac{1}{2} \omega_{\mu} \gamma_{0} \eta+\frac{1}{2} \omega_{\mu}^{a} \gamma_{a} \epsilon^{-}+\frac{1}{2} s_{\mu} \gamma_{0} \epsilon^{+}-r_{1 \mu} \gamma_{0} \eta+b_{\mu} \eta
\end{aligned}
$$

\subsection{Extended Schrödinger supergravity}

As mentioned, our final purpose is to establish the supersymmetric extension of the Schrödinger algebra. The bosonic sector of the Schrödinger algebra extends the Lifshitz algebra (4.1) with non-relativistic special conformal transformations $(K)$ as well as a second central charge $(Z)$. The non-zero commutation relations are given by $[9]$

$$
\begin{aligned}
& {\left[H, G_{a}\right]=-\epsilon_{a b} P^{b}, \quad\left[J, P_{a}\right]=-\epsilon_{a b} P^{b}, \quad\left[J, G_{a}\right]=-\epsilon_{a b} G^{b},} \\
& {\left[P_{a}, G_{b}\right]=\epsilon_{a b} M+\delta_{a b} Y, \quad\left[G_{a}, G_{b}\right]=\epsilon_{a b} S, \quad\left[P_{a}, P_{b}\right]=\epsilon_{a b} Z,} \\
& {\left[D, G_{a}\right]=G_{a}, \quad\left[D, P_{a}\right]=-P_{a}, \quad[D, H]=-2 H,} \\
& {[D, S]=2 S, \quad[D, K]=2 K, \quad[D, Z]=-2 Z,} \\
& {\left[K, P_{a}\right]=-\epsilon_{a b} G^{b}, \quad[K, H]=-D, \quad[K, Y]=S,} \\
& {[K, Z]=2 Y, \quad[H, S]=-2 Y, \quad[H, Y]=-Z,}
\end{aligned}
$$

The corresponding bi-linear form invariant under the extended Schrödinger algebra is given by [9]

$$
\left(P_{a}, G_{b}\right)=\delta_{a b}, \quad(H, S)=-1, \quad(J, M)=-1, \quad(D, Y)=-1, \quad(K, Z)=-1,
$$


Taking the gauge field to be

$$
A_{\mu}=\tau_{\mu} H+e_{\mu}{ }^{a} P_{a}+\omega_{\mu} J+\omega_{\mu}{ }^{a} G_{a}+m_{\mu} M+s_{\mu} S+b_{\mu} D+f_{\mu} K+y_{\mu} Y+z_{\mu} Z,
$$

we can construct the extended Schrödinger gravity action [9]

$$
\begin{aligned}
S=\frac{k}{4 \pi} \int d^{3} x \varepsilon^{\mu \nu \rho}\left(\omega_{\mu}{ }^{a} R_{\nu \rho a}(\right. & P)-2 s_{\mu} R_{\nu \rho}(H)-2 m_{\mu} R_{\nu \rho}(J) \\
& \left.+e_{\mu}{ }^{a} R_{\nu \rho a}(G)-2 y_{\mu} R_{\nu \rho}(D)-2 z_{\mu} R_{\nu \rho}(K)\right) .
\end{aligned}
$$

The supersymmetric extension of the extended Schrödinger algebra requires the inclusion of two new supercharges, $F^{ \pm}$that are both Majorana spinors. With the assistance of the computer algebra program Cadabra [24, 25], we found that the non-vanishing $[B, B]$ commutators of the extended Schrödinger superalgebra are given by (4.13) while the nonvanishing $[B, F]$ commutators are

$$
\begin{aligned}
& {\left[J, Q^{ \pm}\right]=-\frac{1}{2} \gamma_{0} Q^{ \pm},} \\
& {[J, R]=-\frac{1}{2} \gamma_{0} R,} \\
& {\left[S, Q^{+}\right]=-\frac{1}{2} \gamma_{0} R,} \\
& {\left[G_{a}, Q^{+}\right]=-\frac{1}{2} \gamma_{a} Q^{-},} \\
& {\left[J, F^{ \pm}\right]=-\frac{1}{2} \gamma_{0} F^{ \pm},} \\
& {\left[P_{a}, F^{+}\right]=-\frac{1}{2} \epsilon_{a b} \gamma^{b} Q^{-},} \\
& {\left[P_{a}, Q^{-}\right]=-\frac{1}{2} \epsilon_{a b} \gamma^{b} F^{-},} \\
& {\left[G_{a}, Q^{-}\right]=-\frac{1}{2} \gamma_{a} R,} \\
& {\left[U_{1}, R\right]=\gamma_{0} R,} \\
& {\left[U_{1}, F^{ \pm}\right]=\gamma_{0} F^{ \pm},} \\
& {\left[U_{2}, F^{+}\right]=\frac{3}{4} R,} \\
& {\left[H, F^{+}\right]=-Q^{+},} \\
& {\left[U_{1}, Q^{ \pm}\right]= \pm \gamma_{0} Q^{ \pm},} \\
& {\left[D, Q^{+}\right]=-Q^{+},} \\
& {\left[D, F^{ \pm}\right]= \pm F^{ \pm},} \\
& {\left[U_{2}, Q^{+}\right]=-\frac{3}{4} F^{-},} \\
& {\left[K, Q^{+}\right]=F^{+},} \\
& {\left[K, F^{-}\right]=-R \text {, }} \\
& {\left[M, F^{+}\right]=-\frac{1}{4} R,} \\
& {\left[Y, Q^{+}\right]=\frac{1}{4} \gamma_{0} F^{-},} \\
& {[H, R]=F^{-},} \\
& {\left[Z, F^{+}\right]=-\frac{1}{2} \gamma_{0} F^{-},}
\end{aligned}
$$

while the non-vanishing $\{F, F\}$ anti-commutators are given by

$$
\begin{aligned}
& \left\{Q_{\alpha}^{+}, Q_{\beta}^{+}\right\}=\left(\gamma_{0} C^{-1}\right)_{\alpha \beta} H, \\
& \left\{Q_{\alpha}^{+}, Q_{\beta}^{-}\right\}=-\left(\gamma_{a} C^{-1}\right)_{\alpha \beta} P^{a}, \\
& \left\{Q_{\alpha}^{-}, Q_{\beta}^{-}\right\}=\left(\gamma_{0} C^{-1}\right)_{\alpha \beta} M-\left(\gamma_{0} C^{-1}\right)_{\alpha \beta} U_{2}, \\
& \left\{Q_{\alpha}^{+}, R_{\beta}\right\}=\left(\gamma_{0} C^{-1}\right)_{\alpha \beta} M+2 C_{\alpha \beta} Y+\left(\gamma_{0} C^{-1}\right)_{\alpha \beta} U_{2}, \\
& \left\{Q_{\alpha}^{+}, F_{\beta}^{-}\right\}=-2 C_{\alpha \beta} Z, \\
& \left\{F_{\alpha}^{+}, F_{\beta}^{+}\right\}=\left(\gamma_{0} C^{-1}\right)_{\alpha \beta} K, \\
& \left\{F_{\alpha}^{+}, Q_{\beta}^{+}\right\}=-\frac{1}{2}\left(\gamma_{0} C^{-1}\right)_{\alpha \beta} D+\frac{1}{2} C_{\alpha \beta} J+\frac{3}{4} C_{\alpha \beta} U_{1},
\end{aligned}
$$




$$
\begin{aligned}
& \left\{F_{\alpha}^{+}, Q_{\beta}^{-}\right\}=\epsilon_{a b}\left(\gamma^{a} C^{-1}\right)_{\alpha \beta} G^{b}, \\
& \left\{F_{\alpha}^{+}, R_{\beta}\right\}=2 C_{\alpha \beta} S, \\
& \left\{F_{\alpha}^{+}, F_{\beta}^{-}\right\}=\left(\gamma_{0} C^{-1}\right)_{\alpha \beta} M-2 C_{\alpha \beta} Y+\left(\gamma_{0} C^{-1}\right)_{\alpha \beta} U_{2} .
\end{aligned}
$$

The supersymmetric extension of the extended Schrödinger algebra admits the following invariant bilinear forms

$$
\begin{aligned}
& \left(P_{a}, G_{b}\right)=\delta_{a b}, \quad(H, S)=-1, \quad(J, M)=-1, \\
& (D, Y)=-1, \quad(K, Z)=-1, \\
& \left(R_{1}, R_{2}\right)=2, \quad\left(Q_{\alpha}^{+}, R_{\beta}\right)=2 C_{\alpha \beta}, \quad\left(Q_{\alpha}^{-}, Q_{\beta}^{-}\right)=2 C_{\alpha \beta}, \quad\left(F_{\alpha}^{+}, F_{\beta}^{-}\right)=2 C_{\alpha \beta} .
\end{aligned}
$$

Taking the gauge field to be

$$
\begin{aligned}
A_{\mu}= & \tau_{\mu} H+e_{\mu}{ }^{a} P_{a}+\omega_{\mu} J+\omega_{\mu}^{a} G_{a}+m_{\mu} M+s_{\mu} S+b_{\mu} D+f_{\mu} K+y_{\mu} Y+z_{\mu} Z \\
& +r_{1 \mu} U_{1}+r_{2 \mu} U_{2}+\bar{\psi}_{\mu}^{+} Q^{+}+\bar{\psi}_{\mu}^{-} Q^{-}+\bar{\rho}_{\mu} R+\bar{\phi}_{\mu}^{+} F^{+}+\bar{\phi}_{\mu}^{-} F^{-}
\end{aligned}
$$

we obtain the following Chern-Simons action

$$
\begin{aligned}
S=\frac{k}{4 \pi} \int d^{3} x \varepsilon^{\mu \nu \rho} & \left(\omega_{\mu}{ }^{a} R_{\nu \rho a}(P)+e_{\mu}{ }^{a} R_{\nu \rho a}(G)-2 s_{\mu} R_{\nu \rho}(H)\right. \\
& -2 m_{\mu} R_{\nu \rho}(J)-2 y_{\mu} R_{\nu \rho}(D)-2 z_{\mu} R_{\nu \rho}(K)+4 r_{2 \mu} R_{\nu \rho}\left(U_{1}\right) \\
& \left.+4 \bar{\rho}_{\mu} R_{\nu \rho}\left(Q^{+}\right)+2 \bar{\psi}_{\mu}^{-} R_{\nu \rho}\left(Q^{-}\right)+4 \bar{\phi}_{\mu}^{-} R_{\nu \rho}\left(F^{+}\right)\right) .
\end{aligned}
$$

We refer to this action as the extended Schrödinger supergravity. The Schrödinger supercovariant curvatures are given as

$$
\begin{aligned}
R_{\mu \nu}(H) & =2 \partial_{[\mu} \tau_{\nu]}-4 b_{[\mu} \tau_{\nu]}+\bar{\psi}_{\mu}^{+} \gamma_{0} \psi_{\nu}^{-}, \\
R_{\mu \nu}{ }^{a}(P) & =2 \partial_{[\mu} e_{\nu]}^{a}+2 \epsilon^{a b} \omega_{[\mu} e_{\nu] b}-2 \epsilon^{a b} \omega_{[\mu b} \tau_{\nu]}-2 b_{[\mu} e_{\nu]}^{a}-2 \bar{\psi}_{[\mu}^{+} \gamma^{a} \psi_{\nu]}^{-}, \\
R_{\mu \nu}(M) & =2 \partial_{[\mu} m_{\nu]}+2 \epsilon_{a b} \omega_{[\mu}^{a} e_{\nu]}^{b}+2 \bar{\psi}_{[\mu}^{+} \gamma_{0} \rho_{\nu]}+\bar{\psi}_{\mu}^{-} \gamma_{0} \psi_{\nu}^{-}+2 \bar{\phi}_{[\mu}^{+} \gamma_{0} \varphi_{\nu]}, \\
R_{\mu \nu}{ }^{a}(G) & =2 \partial_{[\mu} \omega_{\nu]}^{a}+2 \epsilon^{a b} \omega_{[\mu} \omega_{\nu] b}+2 \epsilon^{a b} f_{[\mu} e_{\nu] b}+2 b_{[\mu} \omega_{\nu]}^{a}-2 \epsilon^{a b} \bar{\phi}_{[\mu}^{+} \gamma_{b} \psi_{\nu]}^{-}, \\
R_{\mu \nu}(J) & =2 \partial_{[\mu} \omega_{\nu]}+\bar{\phi}_{[\mu}^{+} \psi_{\nu]}^{-}, \\
R_{\mu \nu}(S) & =2 \partial_{[\mu} s_{\nu]}+\epsilon^{a b} \omega_{[\mu a} \omega_{\nu] b}+4 b_{[\mu} s_{\nu]}+2 f_{[\mu} y_{\nu]}+4 \bar{\phi}_{[\mu}^{+} \rho_{\nu]}, \\
R_{\mu \nu}(K) & =2 \partial_{[\mu} f_{\nu]}+4 b_{[\mu} f_{\nu]}+\bar{\phi}_{\mu}^{+} \gamma_{0} \phi_{\nu}^{+}, \\
R_{\mu \nu}(D) & =2 \partial_{[\mu} b_{\nu]}+2 \tau_{[\mu} f_{\nu]}-\bar{\phi}_{[\mu}^{+} \gamma_{0} \psi_{\nu]}^{+}, \\
R_{\mu \nu}(Y) & =2 \partial_{[\mu} y_{\nu]}-2 \omega_{[\mu a} e_{\nu]}^{a}+4 f_{[\mu} z_{\nu]}-4 \tau_{[\mu} s_{\nu]}+4 \bar{\psi}_{[\mu}^{+} \rho_{\nu]}-4 \bar{\phi}_{[\mu}^{+} \phi_{\nu]}^{-}, \\
R_{\mu \nu}(Z) & =2 \partial_{[\mu} z_{\nu]}+\epsilon^{a b} e_{[\mu a} e_{\nu] b}-4 b_{[\mu} z_{\nu]}-2 \tau_{[\mu} y_{\nu]}-4 \bar{\psi}_{[\mu}^{+} \varphi_{\nu]},
\end{aligned}
$$




$$
\begin{aligned}
R_{\mu \nu}\left(U_{1}\right)= & 2 \partial_{[\mu} r_{1 \nu]}+\frac{3}{2} \bar{\phi}_{[\mu}^{+} \psi_{\nu]}^{+}, \\
R_{\mu \nu}\left(U_{2}\right)= & 2 \partial_{[\mu} r_{2 \nu]}+2 \bar{\psi}_{[\mu}^{+} \gamma_{0} \rho_{\nu]}-\bar{\psi}_{\mu}^{-} \gamma_{0} \psi_{\nu}^{-}+2 \bar{\phi}_{[\mu}^{+} \gamma_{0} \varphi_{\nu]} \\
R_{\mu \nu}\left(Q^{+}\right)= & 2 \partial_{[\mu} \psi_{\nu]}^{+}+\omega_{[\mu} \gamma_{0} \psi_{\nu]}^{+}-2 \tau_{[\mu} \phi_{\nu]}^{+}-2 b_{[\mu} \psi_{\nu]}^{+}-2 r_{1[\mu} \gamma_{0} \psi_{\nu]}^{+}, \\
R_{\mu \nu}\left(Q^{-}\right)= & 2 \partial_{[\mu} \psi_{\nu]}^{-}+\omega_{[\mu} \gamma_{0} \psi_{\nu]}^{-}+\omega_{[\mu}^{a} \gamma_{a} \psi_{\nu]}^{+}+\epsilon^{a b} e_{[\mu a} \gamma_{b} \phi_{\nu]}^{+}+2 r_{1[\mu} \gamma_{0} \psi_{\nu]}^{-} \\
R_{\mu \nu}(R)= & 2 \partial_{[\mu} \rho_{\nu]}+\omega_{[\mu} \gamma_{0} \rho_{\nu]}+\omega_{[\mu}^{a} \gamma_{a} \psi_{\nu]}^{-}+s_{[\mu} \gamma_{0} \psi_{\nu]}^{+}-2 r_{1[\mu} \gamma_{0} \rho_{\nu]}-\frac{3}{2} r_{2[\mu} \phi_{\nu]}^{+}+2 b_{[\mu} \rho_{\nu]} \\
& -2 f_{[\mu} \phi_{\nu]}^{-}-\frac{1}{2} m_{[\mu} \phi_{\nu]}^{+}-\frac{1}{2} y_{[\mu} \phi_{\nu]}^{+} \\
R_{\mu \nu}\left(F^{+}\right)= & 2 \partial_{[\mu} \phi_{\nu]}^{+}+\omega_{[\mu} \gamma_{0} \phi_{\nu]}^{+}-2 r_{1[\mu} \gamma_{0} \phi_{\nu]}^{+}+2 b_{[\mu} \phi_{\nu]}^{+}+2 f_{[\mu} \psi_{\nu]}^{+} \\
R_{\mu \nu}\left(F^{-}\right)= & 2 \partial_{[\mu} \phi_{\nu]}^{-}+\omega_{[\mu} \gamma_{0} \phi_{\nu]}^{-}-2 r_{1[\mu} \gamma_{0} \phi_{\nu]}^{-}-2 b_{[\mu} \phi_{\nu]}^{-}+\frac{3}{2} r_{2[\mu} \psi_{\nu]}^{+}+2 \tau_{[\mu} \rho_{\nu]} \\
& +\frac{1}{2} m_{[\mu} \psi_{\nu]}^{+}-\frac{1}{2} y_{[\mu} \psi_{\nu]}^{+}+z_{[\mu} \gamma_{0} \phi_{\nu]}^{+} .
\end{aligned}
$$

Finally, the extended Schrödinger supergravity action is invariant under the following transformation rules

$$
\begin{aligned}
\delta \tau_{\mu} & =-\bar{\epsilon}^{+} \gamma_{0} \psi_{\mu}^{+}, \\
\delta e_{\mu}{ }^{a} & =\bar{\epsilon}^{+} \gamma^{a} \psi_{\mu}^{-}+\bar{\epsilon}^{-} \gamma^{a} \psi_{\mu}^{+}, \\
\delta \omega_{\mu}{ }^{a} & =\epsilon^{a b} \bar{\epsilon}^{-} \gamma_{b} \phi_{\mu}^{+}+\epsilon^{a b} \bar{\zeta}^{+} \gamma_{b} \psi_{\mu}^{-}, \\
\delta \omega_{\mu}= & \frac{1}{2} \bar{\epsilon}^{+} \phi_{\mu}^{+}-\frac{1}{2} \bar{\zeta}^{+} \psi_{\mu}, \\
\delta m_{\mu}= & -\bar{\epsilon}^{-} \gamma_{0} \psi_{\mu}^{-}-\bar{\epsilon}^{+} \gamma_{0} \rho_{\mu}-\bar{\eta} \gamma_{0} \psi_{\mu}^{+}-\bar{\zeta}^{+} \gamma_{0} \phi_{\mu}^{-}-\bar{\zeta}^{-} \gamma_{0} \phi_{\mu}^{+}, \\
\delta s_{\mu}= & 2 \bar{\eta} \phi_{\mu}^{+}-2 \bar{\zeta}^{+} \rho_{\mu}, \\
\delta r_{1 \mu}= & \frac{3}{4} \bar{\epsilon}^{+} \phi_{\mu}^{+}-\frac{3}{4} \bar{\zeta}^{+} \psi_{\mu}^{+}, \\
\delta r_{2 \mu}= & \bar{\epsilon} \gamma_{0} \psi_{\mu}^{-}-\bar{\epsilon}^{+} \gamma_{0} \rho_{\mu}-\bar{\eta} \gamma_{0} \psi_{\mu}^{+}-\bar{\zeta}^{+} \gamma_{0} \phi_{\mu}^{-}-\bar{\zeta}^{-} \gamma_{0} \phi_{\mu}^{+}, \\
\delta b_{\mu}= & \frac{1}{2} \bar{\epsilon}^{+} \gamma_{0} \phi_{\mu}^{+}+\frac{1}{2} \bar{\zeta}^{+} \gamma_{0} \psi_{\mu}^{+}, \\
\delta f_{\mu}= & -\bar{\zeta}^{+} \gamma_{0} \phi_{\mu}^{+}, \\
\delta y_{\mu}= & 2 \bar{\eta} \psi_{\mu}^{+}-2 \bar{\epsilon}^{+} \rho_{\mu}-2 \bar{\zeta}^{-} \phi_{\mu}^{+}+2 \bar{\zeta}^{+} \phi_{\mu}^{-}, \\
\delta z_{\mu}= & -2 \bar{\zeta}^{-} \psi_{\mu}^{+}+2 \bar{\epsilon}^{+} \phi_{\mu}^{-}, \\
\delta \psi_{\mu}^{+}= & \partial_{\mu} \epsilon^{+}+\frac{1}{2} \omega_{\mu} \gamma_{0} \epsilon^{+}-\tau_{\mu} \zeta^{+}-b_{\mu} \epsilon^{+}-r_{1 \mu} \gamma_{0} \epsilon^{+}, \\
\delta \psi_{\mu}^{-}= & \partial_{\mu} \epsilon^{-}+\frac{1}{2} \omega_{\mu} \gamma_{0} \epsilon^{-}+\frac{1}{2} \omega_{\mu}^{a} \gamma_{a} \epsilon^{+}+\frac{1}{2} \epsilon^{a b} e_{\mu a} \gamma_{b} \zeta^{+}+r_{1 \mu} \gamma_{0} \epsilon^{-}, \\
\delta \rho_{\mu}= & \partial_{\mu} \eta+\frac{1}{2} \omega_{\mu} \gamma_{0} \eta+\frac{1}{2} \omega_{\mu}^{a} \gamma_{a} \epsilon^{-}+\frac{1}{2} s_{\mu} \gamma_{0} \epsilon^{+}-\frac{1}{4} m_{\mu} \zeta^{+} \\
\delta \phi_{\mu}^{+}= & -\partial_{\mu} \zeta^{+}+\frac{1}{2} \omega_{\mu} \gamma_{0} \zeta^{+}+b_{\mu} \zeta^{+}+f_{\mu} \epsilon^{+}-r_{1 \mu} \gamma_{0} \zeta^{+}, \\
& -r_{1 \mu} \gamma_{0} \eta+\frac{3}{4} r_{2 \mu} \zeta^{+}+b_{\mu} \eta-f_{\mu} \zeta^{-}-\frac{1}{4} y_{\mu} \gamma_{0} \zeta^{+},
\end{aligned}
$$




$$
\begin{aligned}
\delta \phi_{\mu}^{-}= & \partial_{\mu} \zeta^{-}+\frac{1}{2} \omega_{\mu} \gamma_{0} \zeta^{-}+\frac{1}{2} \epsilon_{a b} e_{\mu}{ }^{a} \gamma^{b} \epsilon^{-}+\frac{1}{4} m_{\mu} \epsilon^{+}+r_{1 \mu} \gamma_{0} \zeta^{-} \\
& -\frac{3}{4} r_{2 \mu} \epsilon^{+}+\frac{1}{2} z_{\mu} \gamma_{0} \zeta^{+}-b_{\mu} \zeta^{-}+\tau_{\mu} \eta-\frac{1}{4} y_{\mu} \gamma_{0} \epsilon^{+}
\end{aligned}
$$

where $\zeta^{ \pm}$are the the parameters of the local $F^{ \pm}$transformations, respectively. Due to the map between the Newton-Cartan geometry and Horava-Lifshitz gravity [10], our action (4.21) correspond to a superconformal non-projectable Horava-Lifshitz gravity [9].

\section{Discussion}

In this paper, we establish the supersymmetric extension of the extended Newton-Hooke, Lifshitz and Schrödinger algebras and construct the corresponding Chern-Simons supergravity models. The extended Newton-Hooke superalgebra admits two distinct nondegenerate invariant bi-linear form that gives rise to two different supergravity models with the same equations of motion. These two models are particularly different in terms of the parity of the bosonic actions. In particular, we showed that there is an exotic nonrelativistic model such that parity-even field equations arise from a parity-odd Lagrangian. We then showed that it is possible to improve the extended Bargmann superalgebra with dilatations (without including non-relativistic special conformal symmetry) which we called the extended Lifshitz superalgebra and also established the Chern-Simons extended Lifshitz supergravity action. In the final step, we include the non-relativistic special conformal symmetry and establish the extended Schrödinger superalgebra and the corresponding Chern-Simons extended Schrödinger supergravity action.

We consider our paper as a first step to construct an off-shell formulation for the extended Bargmann supergravity and its matter couplings. Therefore, it would be very useful to study supermultiplet representations of the extended Schrödinger superalgebra. In particular, based on the bosonic construction [18], it is natural to expect that a multiplet with a complex scalar field $\Psi$ as the lowest element can be used to construct a superSchrödinger invariant gravity model. Taking multiple number of such multiplets and gauge fixing the Schrödinger supergravity action would give rise to the supergravity coupling of such a multiplet to off-shell supergravity, which is an important step towards nonrelativistic localization.

The $\mathcal{N}=2$ supergravity models that we established here can be extended to higher number of supercharges. In this case, the extended Bargmann superalgebra with $\mathcal{N}>2$ can be obtained by Lie algebra expansion of the relativistic three-dimensional superalgebras. However, the Lifshitz and the Schrödinger superalgebras cannot be accessed by an InonuWigner contraction or a Lie algebra expansion. In particular, given that the Schrödinger algebra is a particular instance $(\ell=1 / 2)$ of the so called $\ell$-conformal Galilei algebra [35, 36], in which case its $\mathcal{N}>2$ supersymmetric extensions exist [37, 38], it would be very nice to initiate a systematic study to establish extended Schrödinger superalgebras for $\mathcal{N}>2$ supersymmetry. There are also other three-dimensional non-relativistic algebras with an action principle such as the extended Newtonian supergravity [26]. Therefore, such a systematic study would also be helpful to uncover the Schrödinger extension of other threedimensional superalgebras and hopefully towards a catalog of non-relativistic supergravity, 
at least in three dimensions. As a final remark, we would like to point out an interesting relation between the extended Schrödinger algebra and an extended Poincaré algebra. The extended Schrödinger algebra (and extended $\ell$-conformal Galilean algebras) has a hidden relativistic structure such that they can be written in a manifestly $3 \mathrm{~d}$ Lorentz-covariant form [39]. It might of interest to see if the superextension of the Schrödinger superalgebra and the corresponding supergravity theory that we constructed in this paper can also be recast in a manifestly relativistic form.

\section{Acknowledgments}

We thank Eric Bergshoeff for useful comments and suggestions. The authors would like to thank the referee for insightful comments which helped to improve this paper. The work of M.O. is supported in part by TUBITAK grant 118F091. N.O and U.Z. are supported in parts by Istanbul Technical University Research Fund under grant number TDK-201841133.

\section{A Comparison between different notations}

In this paper, we use the conventions of [8]. In what follows we introduce the correspondence between the notations of $[7,8]$ and $[9]$ for reader's convenience.

\begin{tabular}{|c|c|c|}
\hline Bergshoeff et al. [8] & Papageorgiou et al. [7] & Hartong et al. [9] \\
\hline$H$ & $-H$ & $-H$ \\
$P_{a}$ & $-P_{a}$ & $P_{a}$ \\
$G_{a}$ & $\epsilon_{a b} K^{b}$ & $\epsilon_{a b} G^{b}$ \\
$J$ & $J$ & $-J$ \\
$M$ & $-M$ & $-N$ \\
$S$ & $S$ & $S$ \\
\hline
\end{tabular}

\section{B Extended $\mathcal{N}=(2,0)$ Newton-Hooke superalgebra from Lie algebra expansion}

In this appendix, we briefly discuss the Lie algebra expansion origin of the extended Newton-Hooke superalgebra (3.1) and (3.2). Lie algebra expansion, which was originally formulated in [27] and further studied in [28, 29], is a methodology to generate larger Lie algebras starting from a specific one. To obtain larger algebras one first writes down a series expansion for Maurer-Cartan one-forms in powers of an expansion parameter $\lambda$, then inserts these expansions into Maurer-Cartan equation. Finally, the infinite expansions for the Maurer-Cartan form is cut in a consistent way from which one reads off the structure constants of the larger Lie algebra by matching the two sides of the Maurer-Cartan equation in powers of $\lambda$. We refer [30] to readers interested in such three-dimensional bosonic examples in more detail.

The algebra we shall consider here is the $\mathcal{N}=(2,0)$ AdS superalgebra, which consists of the generators of space-time translations $P_{A}$, Lorentz transformations $J_{A}(A=0,1,2)$, 
$R$-symmetry generator $U$ and two supersymmetry generators $Q^{1,2}$ that are both Majorana spinors. The non-zero $[B, B]$ and $[B, F]$ commutation relations for this algebra read $[31,32]$

$$
\begin{aligned}
& {\left[J_{A}, J_{B}\right]=\epsilon_{A B C} J^{C}, \quad\left[J_{A}, P_{B}\right]=\epsilon_{A B C} P^{C}, \quad\left[P_{A}, P_{B}\right]=\frac{1}{\ell^{2}} \epsilon_{A B C} J^{C},} \\
& {\left[J_{A}, Q^{1,2}\right]=\frac{1}{2} \gamma_{A} Q^{1,2}, \quad\left[P_{A}, Q^{1,2}\right]=\frac{1}{2 \ell} \gamma_{A} Q^{1,2}, \quad\left[U, Q^{1,2}\right]= \pm \frac{1}{\ell} Q^{2,1},}
\end{aligned}
$$

while the non-vanising $\{F, F\}$ anti-commutators are given by

$$
\begin{aligned}
& \left\{Q_{\alpha}^{1}, Q_{\beta}^{1}\right\}=2\left(\gamma_{A} C^{-1}\right)_{\alpha \beta} P^{A}+\frac{2}{\ell}\left(\gamma_{A} C^{-1}\right)_{\alpha \beta} J^{A}, \\
& \left\{Q_{\alpha}^{1}, Q_{\beta}^{2}\right\}=2 C_{\alpha \beta} U, \\
& \left\{Q_{\alpha}^{2}, Q_{\beta}^{2}\right\}=2\left(\gamma_{A} C^{-1}\right)_{\alpha \beta} P^{A}+\frac{2}{\ell}\left(\gamma_{A} C^{-1}\right)_{\alpha \beta} J^{A} .
\end{aligned}
$$

To implement the Lie algebra expansion method, we follow the methodology introduced in $[13,30,33,34]$ and decompose the 3-dimensional index $A$ as $A=(0, a)$ in which case the we have

$$
J_{A}=\left(J, G_{a}\right), \quad P_{A}=\left(H, P_{a}\right) .
$$

Furthermore, we choose the following combitation of the fermionic generators,

$$
\tilde{Q}^{ \pm}=\frac{1}{2}\left(Q^{1} \pm \gamma_{0} Q^{2}\right)
$$

We, then, introduce the following gauge fields associated to the generators

$$
\begin{aligned}
& J \rightarrow \Omega, \quad G_{a} \rightarrow \Omega^{a}, \quad H \rightarrow \theta, \quad P_{a} \rightarrow E^{a}, \\
& U \rightarrow A, \quad \tilde{Q}^{+} \rightarrow \Psi^{+}, \quad \tilde{Q}^{-} \rightarrow \Psi^{-}
\end{aligned}
$$

The expansion of the gauge fields are given by

$$
\begin{aligned}
& \Omega=\omega+\lambda^{2} s, \quad E^{a}=\lambda e^{a}, \quad \theta=\tau+\lambda^{2} m, \quad \Omega^{a}=\lambda \omega^{a}, \\
& A=r_{1}+\lambda^{2} r_{2}, \quad \Psi^{+}=\psi^{+}+\lambda^{2} \rho, \quad \Psi^{-}=\lambda \psi^{-} \text {. }
\end{aligned}
$$

Using these expansions into the prescription given above, we precisely arrive at the extended Newton-Hooke superalgebra (3.1) and (3.2).

Open Access. This article is distributed under the terms of the Creative Commons Attribution License (CC-BY 4.0), which permits any use, distribution and reproduction in any medium, provided the original author(s) and source are credited.

\section{References}

[1] E. Cartan, Sur les variétés à connexion affine et la théorie de la relativité généralisée. (première partie), Annales Sci. Ecole Norm. Sup. 40 (1923) 325.

[2] E. Cartan, Sur les variétés à connexion affine et la théorie de la relativité généralisée. (première partie) (Suite), Annales Sci. Ecole Norm. Sup. 41 (1924) 1. 
[3] A. Trautman, Sur la theorie newtonienne de la gravitation, Compt. Rend. Acad. Sci. Paris 247 (1963) 617.

[4] J. Ehlers, Über den Newtonschen Grenzwert, in Grundlagen-probleme der modernen Physik, J. Nitsch et al. eds., Bibliographisches Institut Mannheim, Germany (1981).

[5] R. Andringa, E. Bergshoeff, S. Panda and M. de Roo, Newtonian gravity and the Bargmann algebra, Class. Quant. Grav. 28 (2011) 105011 [arXiv:1011.1145] [INSPIRE].

[6] E. Witten, $(2+1)$-dimensional gravity as an exactly soluble system, Nucl. Phys. B 311 (1988) 46 [INSPIRE].

[7] G. Papageorgiou and B.J. Schroers, A Chern-Simons approach to Galilean quantum gravity in 2+1 dimensions, JHEP 11 (2009) 009 [arXiv:0907.2880] [INSPIRE].

[8] E.A. Bergshoeff and J. Rosseel, Three-dimensional extended Bargmann supergravity, Phys. Rev. Lett. 116 (2016) 251601 [arXiv:1604.08042]. .

[9] J. Hartong, Y. Lei and N.A. Obers, Nonrelativistic Chern-Simons theories and three-dimensional Hor̆ava-Lifshitz gravity, Phys. Rev. D 94 (2016) 065027 [arXiv: 1604.08054].

[10] J. Hartong and N.A. Obers, Hořava-Lifshitz gravity from dynamical Newton-Cartan geometry, JHEP 07 (2015) 155 [arXiv: 1504.07461] [INSPIRE].

[11] V. Pestun, Localization of gauge theory on a four-sphere and supersymmetric Wilson loops, Commun. Math. Phys. 313 (2012) 71 [arXiv:0712.2824] [InSPIRE].

[12] G. Festuccia and N. Seiberg, Rigid supersymmetric theories in curved superspace, JHEP 06 (2011) 114 [arXiv:1105.0689] [INSPIRE].

[13] J.A. de Azcárraga, D. Gútiez and J.M. Izquierdo, Extended D $=3$ Bargmann supergravity from a Lie algebra expansion, arXiv:1904.12786 [INSPIRE].

[14] M. Kaku, P.K. Townsend and P. van Nieuwenhuizen, Properties of conformal supergravity, Phys. Rev. D 17 (1978) 3179 [inSPIRE].

[15] M. Kaku and P.K. Townsend, Poincare supergravity as broken superconformal gravity, Phys. Lett. B 76 (1978) 54.

[16] S. Ferrara, M. Kaku, P.K. Townsend and P. van Nieuwenhuizen, Gauging the graded conformal group with unitary internal symmetries, Nucl. Phys. B 129 (1977) 125 [INSPIRE].

[17] M. Kaku, P.K. Townsend and P. van Nieuwenhuizen, Gauge theory of the conformal and superconformal group, Phys. Lett. B 69 (1977) 304.

[18] H.R. Afshar et al., A Schrödinger approach to Newton-Cartan and Hořava-Lifshitz gravities, JHEP 04 (2016) 145 [arXiv:1512.06277] [INSPIRE].

[19] E. Bergshoeff, J. Rosseel and T. Zojer, Newton-Cartan supergravity with torsion and Schrödinger supergravity, JHEP 11 (2015) 180 [arXiv: 1509.04527] [INSPIRE].

[20] E. Joung and W. Li, Nonrelativistic limits of colored gravity in three dimensions, Phys. Rev. D 97 (2018) 105020 [arXiv: 1801.10143] [INSPIRE].

[21] L. Avilés et al., Non-relativistic Maxwell Chern-Simons gravity, JHEP 05 (2018) 047 [arXiv: 1802.08453] [INSPIRE].

[22] P.K. Townsend and B. Zhang, Thermodynamics of "exotic" Bañados-Teitelboim-Zanelli black holes, Phys. Rev. Lett. 110 (2013) 241302 [arXiv:1302.3874] [INSPIRE]. 
[23] R. Andringa, E.A. Bergshoeff, J. Rosseel and E. Sezgin, 3D Newton-Cartan supergravity, Class. Quant. Grav. 30 (2013) 205005 [arXiv:1305.6737] [INSPIRE].

[24] K. Peeters, A field-theory motivated approach to symbolic computer algebra, Comput. Phys. Commun. 176 (2007) 550 [cs/0608005] [INSPIRE].

[25] K. Peeters, Introducing Cadabra: a symbolic computer algebra system for field theory problems, hep-th/0701238 [INSPIRE].

[26] N. Ozdemir, M. Ozkan, O. Tunca and U. Zorba, Three-dimensional extended newtonian (super)gravity, JHEP 05 (2019) 130 [arXiv: 1903.09377] [INSPIRE].

[27] M. Hatsuda and M. Sakaguchi, Wess-Zumino term for the AdS superstring and generalized Inonu-Wigner contraction, Prog. Theor. Phys. 109 (2003) 853 [hep-th/0106114] [INSPIRE].

[28] J.A. de Azcarraga, J.M. Izquierdo, M. Picón and O. Varela, Generating Lie and gauge free differential (super)algebras by expanding Maurer-Cartan forms and Chern-Simons supergravity, Nucl. Phys. B 662 (2003) 185 [hep-th/0212347] [INSPIRE].

[29] J.A. de Azcarraga, J.M. Izquierdo, M. Picón and O. Varela, Expansions of algebras and superalgebras and some applications, Int. J. Theor. Phys. 46 (2007) 2738 [hep-th/0703017] [INSPIRE].

[30] E. Bergshoeff, J.M. Izquierdo, T. Ortín and L. Romano, Lie algebra expansions and actions for non-relativistic gravity, JHEP 08 (2019) 048 [arXiv: 1904.08304] [INSPIRE].

[31] A. Achucarro and P.K. Townsend, A Chern-Simons action for three-dimensional Anti-de Sitter supergravity theories, Phys. Lett. B 180 (1986) 89 [InSPIRE].

[32] A. Achucarro and P.K. Townsend, Extended supergravities in $d=(2+1)$ as Chern-Simons theories, Phys. Lett. B 229 (1989) 383 [InSPIRE].

[33] L. Romano, Non-relativistic four dimensional p-brane supersymmetric theories and Lie algebra expansion, arXiv: 1906.08220 [INSPIRE].

[34] D.M. Peñafiel and P. Salgado-ReboLledó, Non-relativistic symmetries in three space-time dimensions and the Nappi-Witten algebra, Phys. Lett. B 798 (2019) 135005 [arXiv: 1906.02161] [INSPIRE].

[35] J. Negro, M. del Olmo and A. Rodriguez-Marco, Nonrelativistic conformal groups, J. Math. Phys. 38 (1997) 3786.

[36] M. Henkel, Local scale invariance and strongly anisotropic equilibrium critical systems, Phys. Rev. Lett. 78 (1997) 1940 [cond-mat/9610174] [INSPIRE].

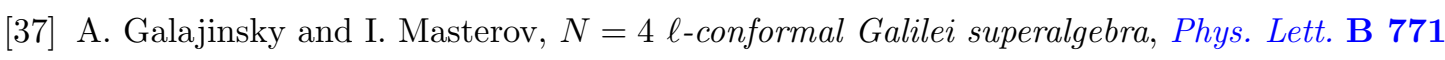
(2017) 401 [arXiv: 1705. 02814] [INSPIRE].

[38] A. Galajinsky and S. Krivonos, $N=4 \ell$-conformal Galilei superalgebras inspired by $D(2,1 ; \alpha)$ supermultiplets, JHEP 09 (2017) 131 [arXiv:1706.08300] [INSPIRE].

[39] D. Chernyavsky and D. Sorokin, Three-dimensional (higher-spin) gravities with extended Schrödinger and l-conformal Galilean symmetries, JHEP 07 (2019) 156 [arXiv:1905.13154] [INSPIRE]. 